Thorax (1964), 19, 383

\title{
Lung transplantation: technical problems
}

\author{
J. BOR RIE AND I. LICHTER
}

From the Department of Surgery, University of Otago, Dunedin, New Zealand

The biological problems involved in successful organ transplantation are well known and have been reviewed by Gowans (1962) and by Howard and Michie (1963). Besides these there are many technical difficulties.

These may be studied experimentally by lung excision and re-implantation in the same animal, or by lung transplantation from unrelated or related animals. Further, the tolerance time of lungs to anoxia can be evaluated.

\section{LUNG EXCISION AND RE-IMPLANTATION}

In 1951, Juvenelle, Citret, Wiles, and Stewart reported a six-month survivor from a series of dogs subjected to pneumonectomy and reimplantation of the vessels and bronchi of the right lung. In 1952, Neptune, Redondo, and Bailey had a successful re-implant in dogs one year after operation. In 1959, Huggins reported successful excision and re-implantation of the left lower lobe of the dog lung. In 1963, Alican and Hardy reported 33 long-term survivors in dogs in which either the right or the left lung had been re-implanted.

In studying these survivors further by contralateral pneumonectomy, pneumonectomy and re-implantation, pulmonary artery ligation or multiple lobectomies, they drew the following conclusions :

(1) Pulmonary oedema may frequently develop when the blood-flow through the re-implanted lung is suddenly increased.

(2) The altered pattern of respiration due to the absence of reflexes originating in the lungs may not be compatible with long-term survival, or at least the animal without these reflexes is at a definite disadvantage as far as ventilatory adjustments are concerned.

(3) In future lung homotransplantations, the preservation of an appropriate amount of the recipient's own lung tissue to prevent alteration in the normal respiratory pattern and to decrease the chances of pulmonary oedema would be of serious concern.
EXCISION AND RE-IMPLANTATION OF LUNGS IN SHEEP

Since 1957 the technical steps that have to be taken in transplanting lungs have been studied in the Department of Surgery, University of Otago. The purpose of this paper is to describe the techniques used and the problems encountered.

Initially, because of the homograft reaction, the stages in a suitable technique were established by completely excising and re-implanting a lung of the same animal, checking the efficacy of the procedure by differential lung function studies of oxygen uptake before operation, immediately afterwards, and again after two months. It was felt that the post-operative ability of a lung to absorb oxygen as determined by differential bronchospirometry gave the best index of the success of the experiment. Histological studies were confirmatory. Further, such a procedure could give a completely denervated lung, which is ideal for physiological study of the nature of respiratory reflexes.

The sheep was chosen as the experimental animal, and the left lung was used throughout. The right lung was deemed unsuitable because of its tracheal bronchus to the cephalic lobe.

LUNG FUNCTION: DIFFERENTIAL BRONCHOSPIROMETRY For experimental ease, readily available standard equipment was used. Though the trachea of a sheep is too long to use a normal Carlens endobronchial tube inserted through the mouth, pre- and post-operative bronchospirometry as a test of lung function by oxygen uptake can be satisfactorily performed through a temporary tracheostomy (Borrie and Montgomerie, 1958a and b).

Animals weighing from 40 to $45 \mathrm{~kg}$. were used. After premedication with morphine $(15 \mathrm{mg}$.) and atropine (1.2 mg.), general anaesthesia was induced with pentobarbitone $(64 \mathrm{mg} . / 8 \mathrm{~kg}$. body weight intravenously), supplemented as required with open ether. The trachea was exposed below the thyroid gland and incised transversely. A no. 37 or 39 Carlens tube was inserted, and, with the animal still lightly anaesthetized and breathing regularly, pul- 
monary ventilation and oxygen uptake were recorded with a Palmer twin-recording bronchospirometer. ${ }^{1}$

Once a satisfactory record had been obtained, the Carlens tube was removed, a size 8 cuffed Magill endotracheal tube was inserted through the tracheostomy, and anaesthesia was continued by attaching the tube to a Palmer anaesthetic pump which had been set to the appropriate tidal volume as determined from a weight-tidal volume chart (Borrie and Mitchell, 1960).

After completing the experimental procedure, the chest wall was closed over water-seal drainage, and post-operative differential bronchospirometry was performed by removing the Magill tube and reinserting the Carlens tube. Finally, the tracheostome was closed with interrupted sutures placed through the peritracheal fascia. It had previously been shown that simple transverse tracheal wounds in the sheep heal rapidly without stricture formation (Borrie, 1957). Each animal also received penicillin. 1 million units, and streptomycin. $1 \mathrm{~g}$. daily for seven days after operation.

Two months after operation lung function was reassessed by repeating the procedure. Thereafter the animal was killed, and symmetrical specimens from the two lungs were taken to determine the normal sheep lung histology.

TECHNIQUE OF LEFT LUNG EXCISION AND RE-IMPLANTATION After several pilot experiments total lung excision and re-implantation was performed (Borrie and Montogomerie, 1958a and b).

Excision After differential bronchospirometry a standard left thoracotomy was performed through the fifth intercostal space (Fig. 1). The pulmonary liga-

1 C. F. Palmer \& Co., Effra Road, London, S.W.2. ment was divided to avoid injuring the lung. The pericardial cavity was opened in front of the pulmonary veins, and the left phrenic nerve was mobilized and protected with stay sutures passed through

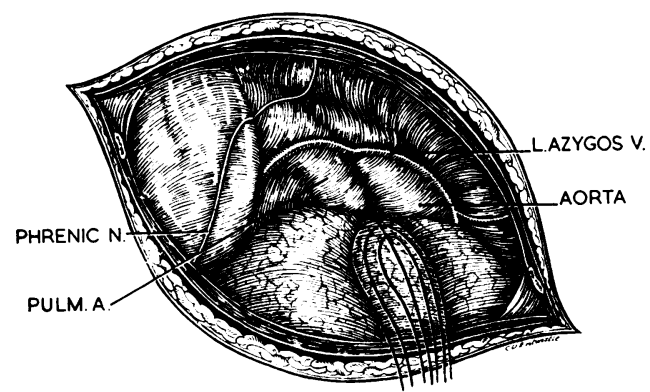

FIG. 1. View of the medial wall of the left pleural cavity of a sheep via a left fifth intercostal thoracotomy.

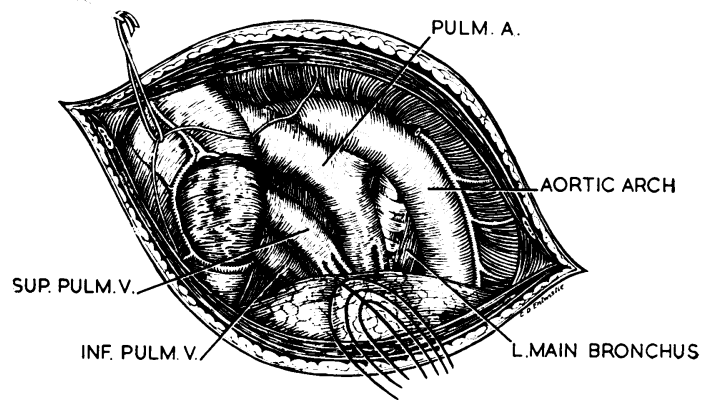

FIG. 2. View after incising the mediastinal pleura and ligating the left azygos vein. (a)

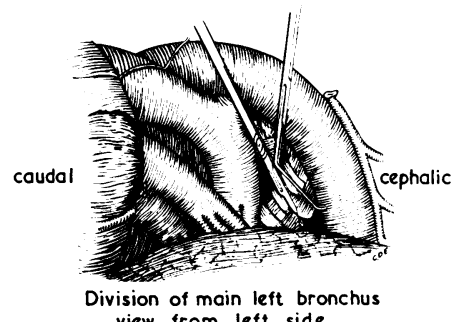

view from left side (b)

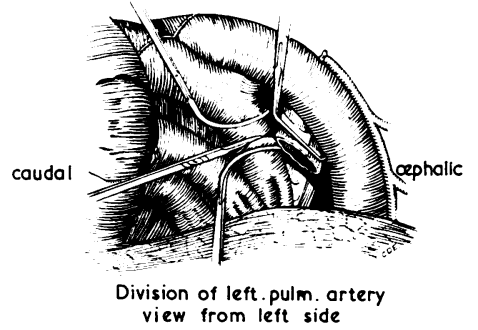

(c)

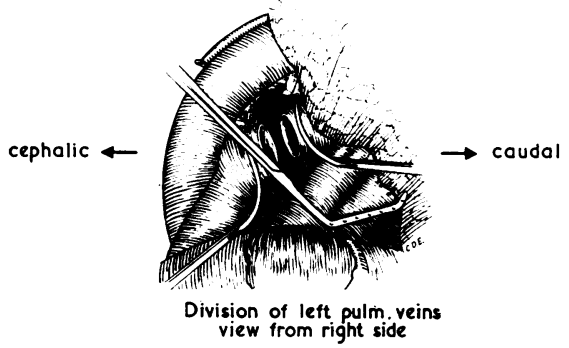

FIG. 3. Technique of lung excision. 
the pericardial flaps. The hemiazygos vein, a constant feature of the sheep, was next ligated and divided as it entered the coronary sinus (Fig. 2).

The left main bronchus was isolated from the level of the carina to its upper lobar branch, a $2-\mathrm{cm}$. length, carefully clamped at the carina, and divided (Fig. 3a).

The left pulmonary artery was dissected to its origin, doubly clamped with Potts spoon-shaped multi-toothed arterial clamps, and divided (Fig. 3b).

The upper and lower left pulmonary veins were finally isolated, clamped with a toothed bronchus clamp, and divided, thus excising the lung (Fig. 3c). After excision of the lung these venous openings into the heart were closed in two layers with continuous silk sutures. The artery of the excised lung was immediately perfused with $6 \%$ dextran in $0.9 \%$ saline at $4^{\circ} \mathrm{C}$. and $15 \mathrm{~mm}$. $\mathrm{Hg}$ pressure.

RE-IMPLANTATION It was initially assumed to be technically easier and more satisfactory to anastomose the common left pulmonary venous stump to a fresh opening in the left auricle than to rejoin it to the pulmonary venous stumps. A suitable length of auricle was therefore lifted in a Brock auricular clamp and incised, and a one-layer vascular anastomosis was made with Blalock everting sutures between the pulmonary veins and the heart. Later experience, however, with re-implantation of the lungs showed it was difficult in sheep to get an adequate cuff of atrium containing both left pulmonary veins for this re-implantation experiment, and that it was desirable to resuture the veins to their original site.

(a)

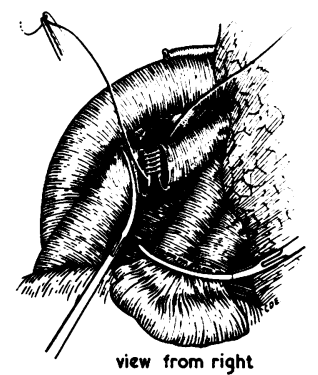

(b)

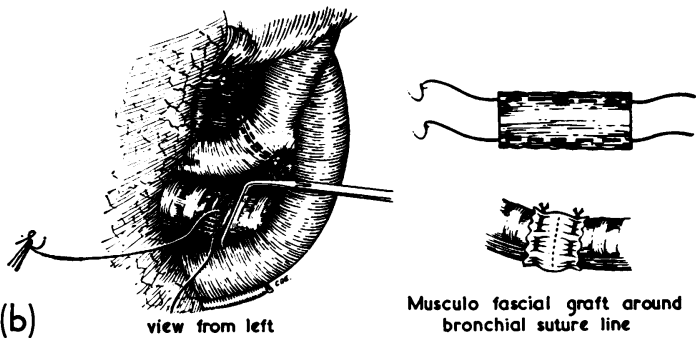

FIG. 4. Technique of anastomosis of (a) the left pulmonary artery, and (b) the left main bronchus. The veins have already been anastomosed to the left atrial appendix.
The pulmonary artery was similarly resutured (Fig. $4 a$ ), and the clamps on the artery and vein were released. The average time taken to complete this vascular stage was one and a quarter hours.

Thereafter, suture of the left main bronchus completed the re-implantation (Fig. 4b). Additional airseal was secured by a free fascial graft collar around the bronchial suture line, taken from the serratus anterior muscle. The chest wall was closed with water-seal drainage, and further spirometry was performed to assess the oxygen uptake per lung.

Results None of the 10 sheep in the original series survived more than six days; deaths within 24 hours were due to tension pneumothorax (1), operative haemorrhage (2), and bronchial obstruction (3), and later deaths were due to a slowly developing thrombosis within the pulmonary vascular bed, producing pulmonary infarction.

Of five animals investigated by immediate postoperative differential bronchospirometry, two showed ventilatory and oxygen absorbing function similar to the values found pre-operatively (Fig. 5a and $b$ ), and one showed a latent function made apparent when the right lung was occluded (Fig. 5c and $\mathrm{d}$ ).

Comment These experiments showed that it was possible to restore ventilatory and oxygen absorbing function, at least temporarily, after excising and re-implanting a lung in sheep, and raised the hope that longer survivals could successfully be achieved. Death was most commonly due to pulmonary infarction. As this could have arisen in the pulmonary or bronchial arteries, or the veins, its basic cause and method of prevention were further investigated by studying separately the effects of dividing and resuturing the four major components of the lung hilum. Each of these problems was checked in sets of six experiments controlled by lung function studies before, immediately after, and up to three months after operation.

1. BRONCHIAL ARTERY DIVISION AND LUNG FUNCTION In the earlier 'total' experiment of lung excision and re-implantation the minute left bronchial artery was merely ligated. The effect of this ligation on subsequent lung function was therefore first investigated.

Technique After differential bronchospirometry the hilum of the left lung was dissected, the left main bronchus isolated, the bronchial artery divided and ligated, and the thoracotomy closed. Differential bronchospirometry was then repeated, and again two months later, after which the sheep were killed and the lungs were examined histologically (Fig. 6). 


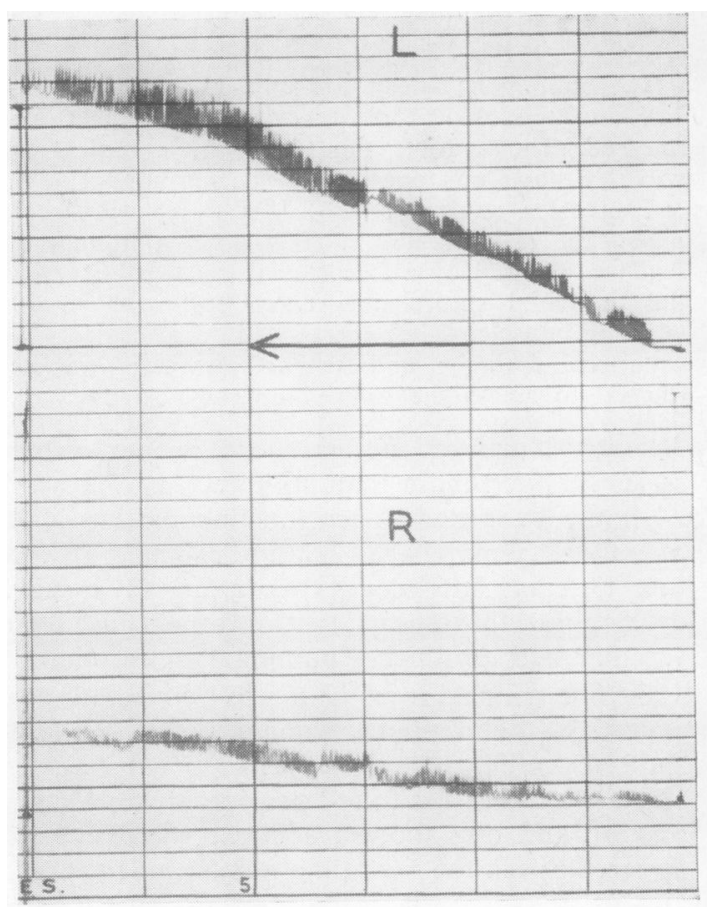

(a)

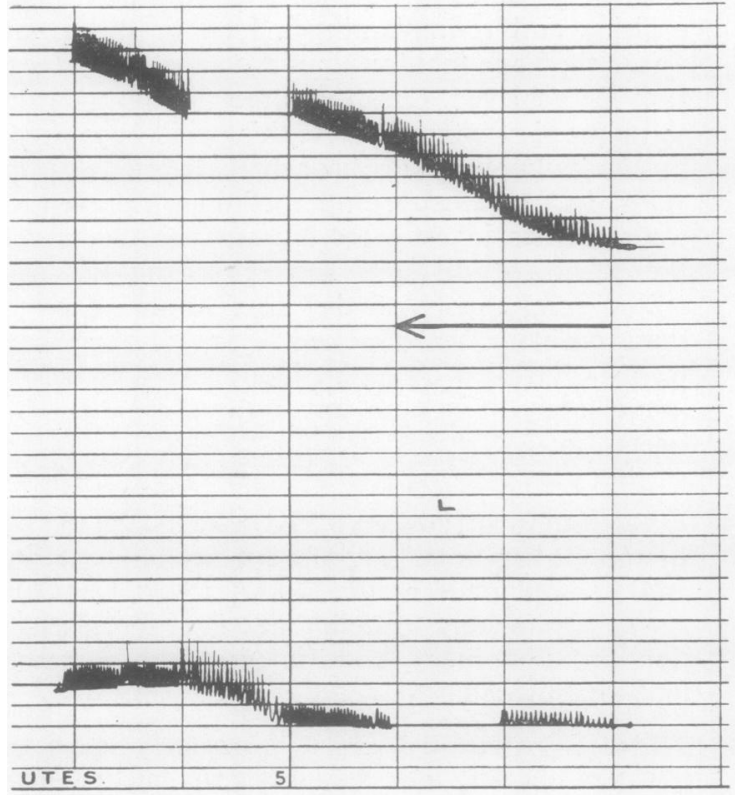

(c)

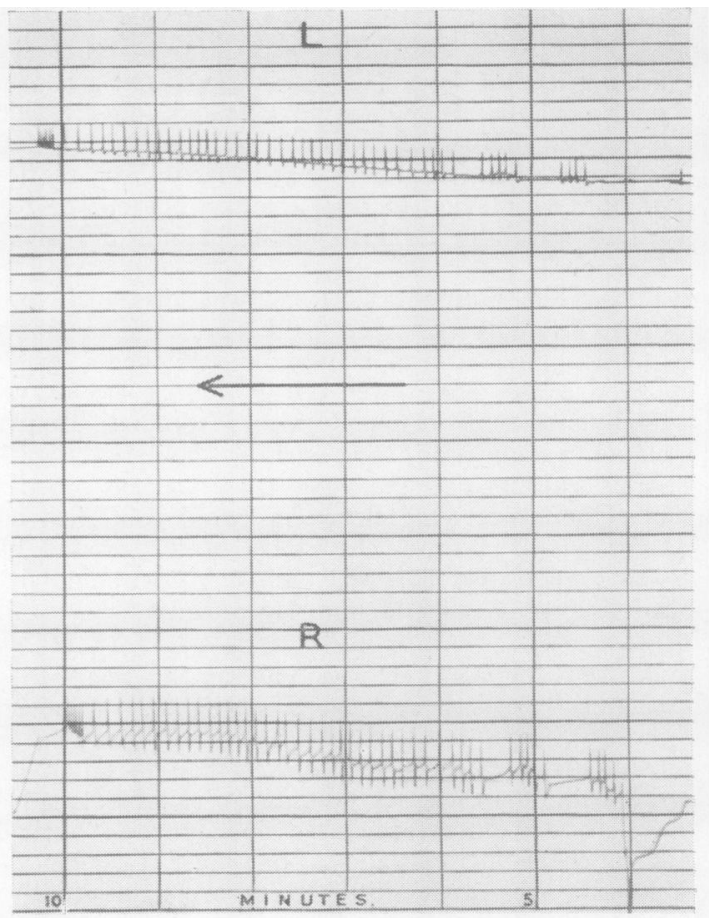

(b)

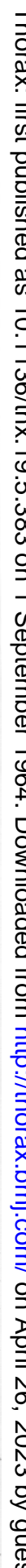

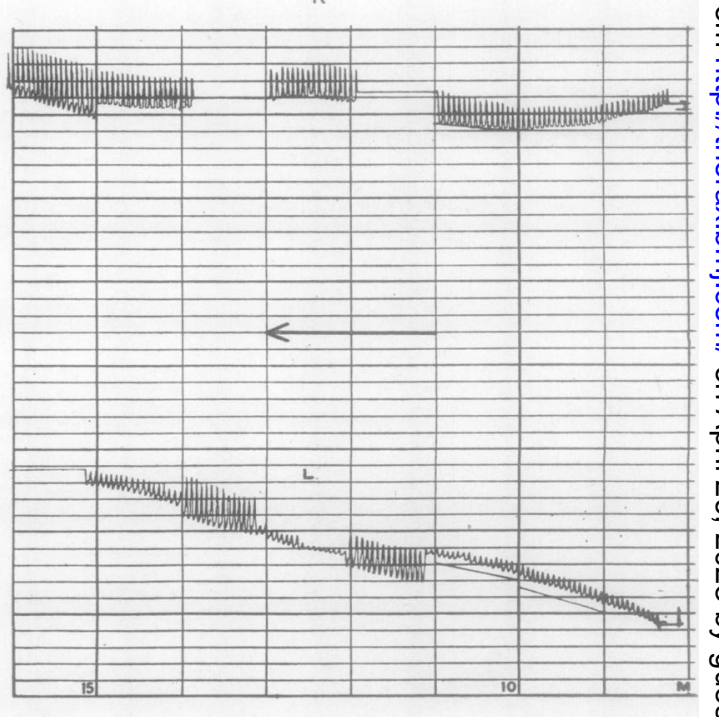

(d)

FIG. 5. Differential spirograms of sheep showing oxygen uptake of the left lung (a) before and (b) immediately after total excision and re-implantation. (These spirograms are read from right to left.) Similar spirograms taken (c) pre-operatively and $(d)$ post-operatively show a latent function in the left lung made more obvious when the right lung is temporarily occluded for minute intervals. 


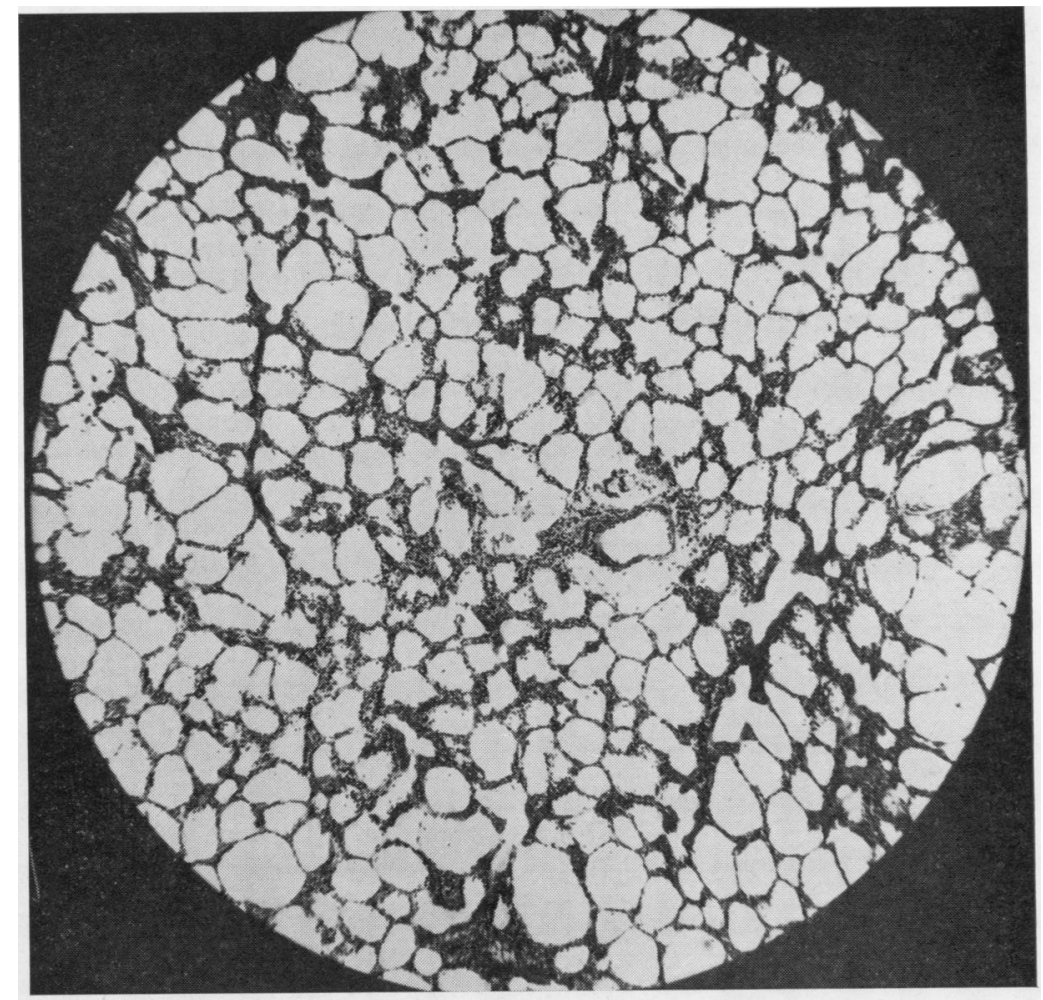

FIG. 6. Histological pattern of (above) the right and (below) the left lung two months after division of the left bronchial artery. 
Results All six animals survived operation. Preoperative spirometry established the normal oxygen uptake of each lung. Immediately after operation the total oxygen uptake was reduced, but the left lung/right lung oxygen absorption ratio remained unchanged in all but one animal. Two months later there was still the same proportionate oxygen uptake by the left lung, and the animals remained healthy and thriving (Fig. 7).

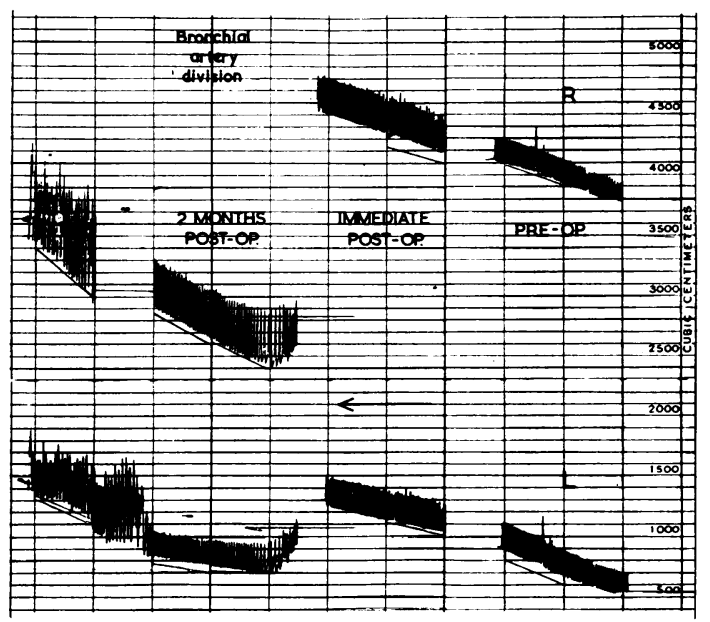

FIG. 7. Differential spirograms before, immediately after, and two months after operation, showing satisfactory oxygen uptake in the left lung after division of the bronchial artery.

It is concluded that in the normal sheep the rate of oxygen uptake is greater in the right than in the left lung, and that division and ligation of the bronchial artery has no measurable effect on the proportionate oxygen uptake of the left lung either immediately after or within two months of operation (Borrie, Campbell, and Fulton, 1958).

2. PULMONARY ARTERY DIVISION, RESUTURE, AND LUNG FUNCTION Normal pulmonary vascular pressures in the sheep were measured. They were: pulmonary artery, $20 / 14 \mathrm{~mm}$. $\mathrm{Hg}$; pulmonary vein, $5 / 2.25 \mathrm{~mm}$. $\mathrm{Hg}$; and left atriu, $7.5 \mathrm{~mm}$. $\mathrm{Hg}$. When both pulmonary veins were clamped, the pressure on the pulmonary side rose and oscillated between 17 and $15 \mathrm{~mm}$. $\mathrm{Hg}$.

Technique After routine anaesthesia, differential bronchospirometry, and left thoracotomy, the left pulmonary artery was isolated, doubly clamped with Potts' arterial clamps, divided, and then resutured using the Blalock everting technique with 4-0 silk sutures. Anticoagulants were not used. Post-operative bronchospirometry was performed, and the wounds were closed. Three months later, after further differ- ential bronchospirometry, the lungs and pulmonar arteries were examined.

Results All eight animals survived operation, bu two died in the early post-operative period, from sputum retention, and from empyema.

Immediate and late post-operative broncho $\overrightarrow{0}$ spirometry showed that the proportionate oxygen uptake of the left lung was unaltered by the opera tion (Fig. 8). Necropsy confirmed patency of thet left pulmonary artery in all cases and reveale a soundly healed anastomosis with no evidenc $\oplus$ of intravascular clotting or obstruction (Fig. 9 s The histological picture was normal in the tese lung.
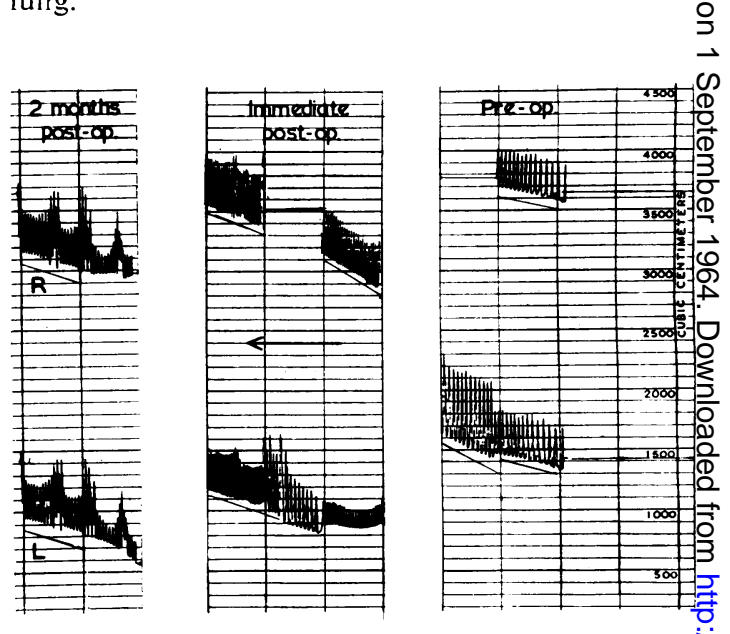

FIG. 8. Differential bronchospirometry where the lef pulmonary artery was divided and rejoined: tested before immediately after, and two months after operation.

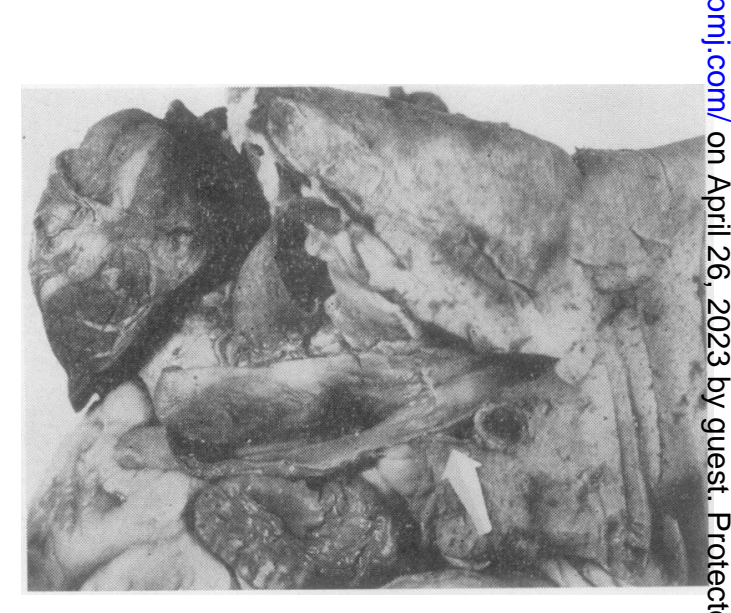

FIG. 9. The left pulmonary artery showing the site of division and resuture and no occlusion or narrowing of the lumen two months after operation. 
It is concluded that division and resuture of the left pulmonary artery is regularly feasible without interfering with the ability of the left lung to absorb oxygen. These results further suggested that the initial problem of intravascular clotting after lung excision and re-implantation did not lie on the arterial side (Borrie and Fulton, 1958).

\section{LEFT MAIN BRONCHUS DIVISION, RESUTURE, AND LUNG FUNCTION}

Technique Once again, after anaesthesia, differential bronchospirometry, and left thoracotomy, both the right and left main bronchi were isolated in the mediastinum. The left main bronchus was dissected free from the carina down to its upper lobe bronchus. It was lightly clamped on the proximal side only at carinal level with a Satinsky clamp, and divided in the middle of the exposed segment. Resuture was performed with a continuous 0000 silk suture, using the Blalock technique.

An interrupted locking stitch was also used after every three bites of the continuous suture. After the resuture had been completed, the clamp on the bronchus was released, and the lung was inflated. A fascial collar $2 \mathrm{~cm}$. wide was again cut from the serratus anterior muscle, threaded round the line of anastomosis, and sutured into place. This achieved air-tight bronchial closure. The chest was closed by standard technique with tube drainage. Post-operative bronchoscopy and differential spirometry were next performed, and the tracheostome was closed.

Two months later, under general anaesthesia, bronchoscopy was performed, followed by differential bronchospirometry. The lungs and bronchial suture line were later examined.

Results The experiment was performed on six sheep without death. In the first animal, in which

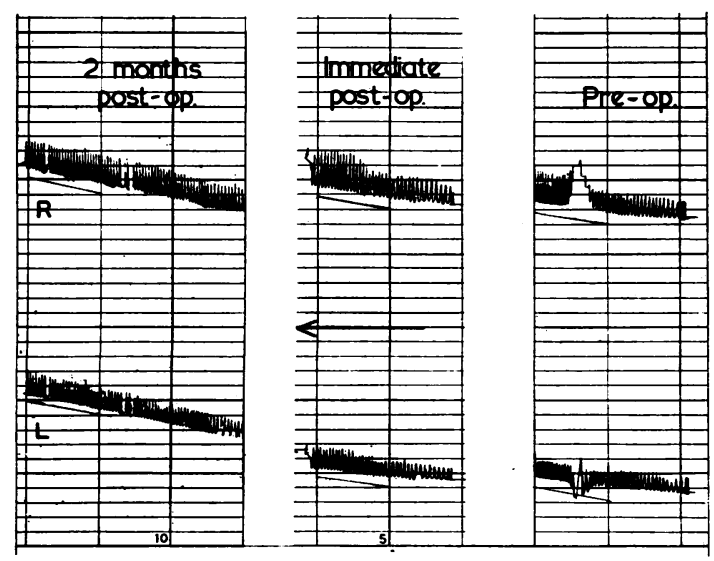

FIG. 10. Differential spirogram showing normal oxygen uptake in the left lung before, after, and two months after division and resuture of the left main bronchus.

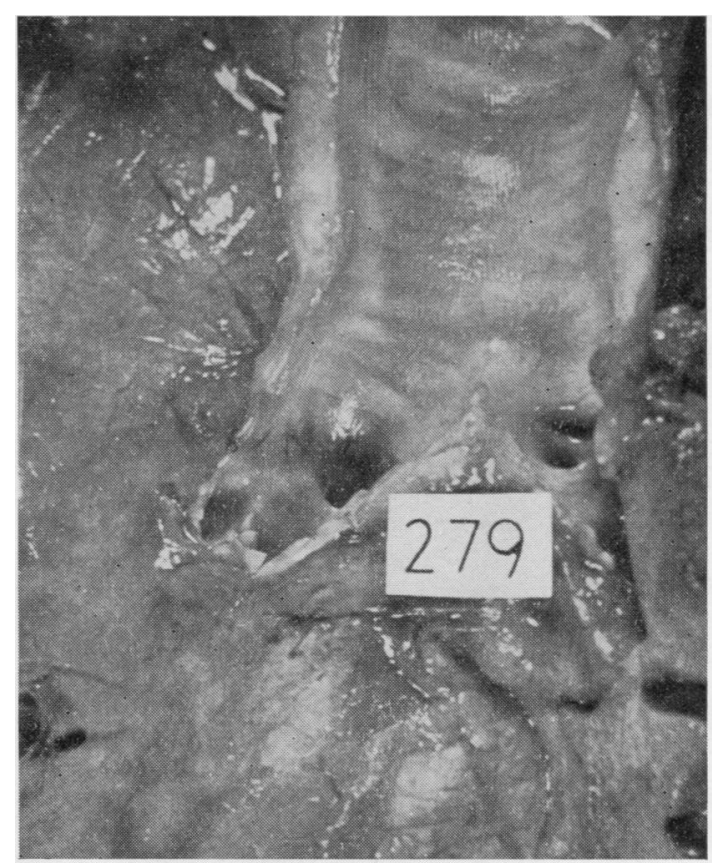

FIG. 11. View of the lower trachea, carina, and main bronchi-from behind-showing suturing just proximal to the left upper lobe bronchial orifice (left-hand side) and no bronchostenosis.

the technique was being established, there was a residual left bronchial stricture, but some oxygen uptake of the left lung. The remaining five animals two months after operation showed normal oxygen uptake in the test lung (Fig. 10). Necropsy showed a normal bronchial lumen with no suggestion of narrowing at the line of bronchial resuture (Fig. 11). The histology of the five lungs was also normal (Fig. 12).

It is concluded that this technique gives a satisfactory bronchial anastomosis after bronchial division, without air leak, stricture formation or adverse effest on lung function up to two months after operation (Borrie and Foster, 1959a).

4. DIVISION AND RESUTURE OF PULMONARY VEINS In the early reported successes in dogs of pulmonary re-implantation and transplantation, a left atrial cuff containing the orifices of the two pulmonary veins had usually been re-anastomosed to a new opening fashioned in the left atrial appendix.

In sheep, however, with a small, squat left atrium, a mechanical problem arose. For, even when using anticoagulants, the short length of 


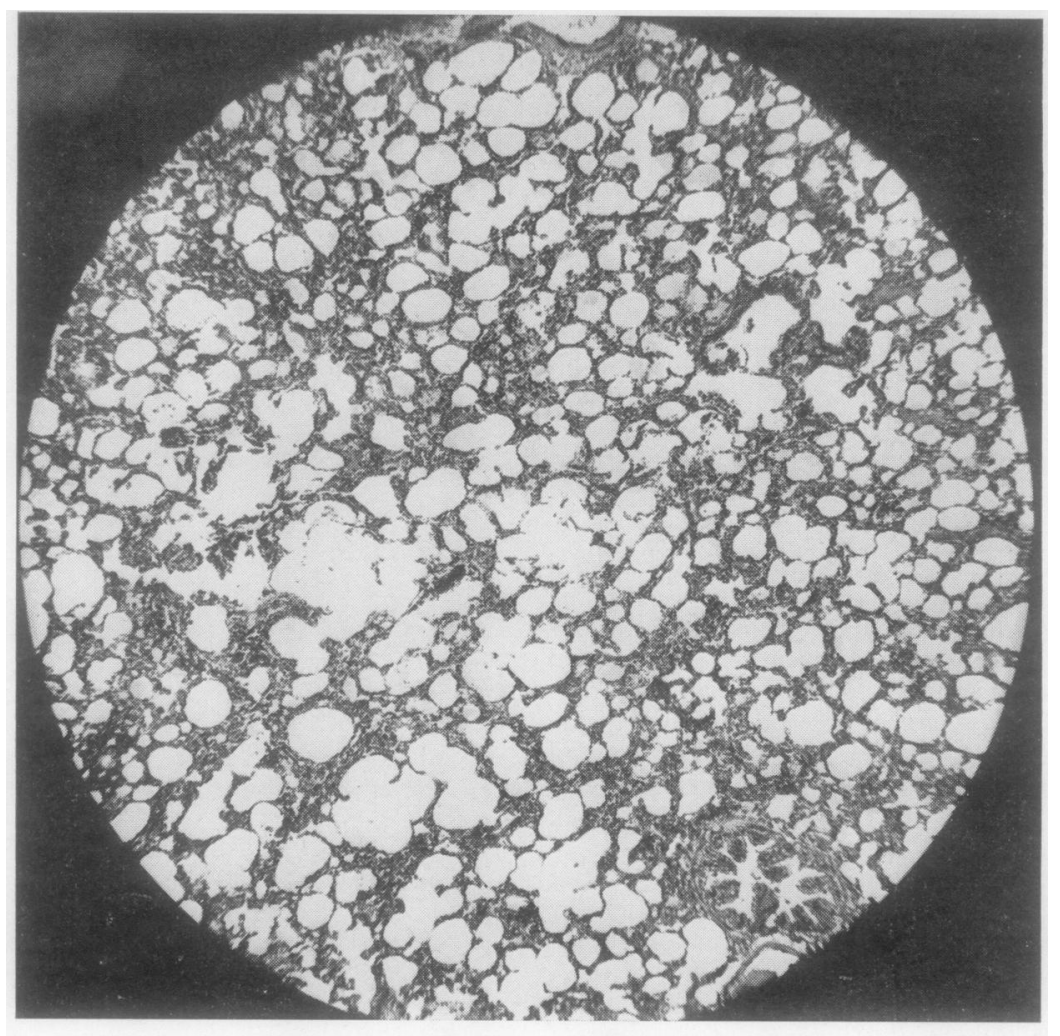

FIG. 12. Histological pattern of (above) the right, and (below) the left lung two months after division and resuture of the left main bronchus. 
pulmonary veins available for anastomosis to the atrial appendix, and consequent stitching, caused collapse and mechanical occlusion of the venous lumen, leading to the rapid onset of venous thrombosis with pulmonary infarction (Borrie and Foster, 1959b).

Further, whereas venous autografts regularly remained patent, intravenous thrombosis regularly occurred after the experimental use of freeze-dried venous homografts.

In the further experiments, therefore, in reimplantation of lungs in sheep, the pulmonary veins were re-implanted into their normal position. The procedure was greatly helped with a Sellors atrial clamp.

Technique After differential spirometry and left thoracotomy the lung hilum was dissected. The left bronchial artery was ligated, the pericardium incised, the pulmonary artery encircled with a tape, and both left pulmonary veins dissected. Twelve thousand i.u. of heparin were given, and then the circulation to the left lung was interrupted by tightening the arterial tape and clamping both veins well on to the left atrium with a Sellors atrial clamp. Both pulmonary veins were divided and in turn resutured, using everting sutures of 0000 silk with every second stitch locked.
Pulmonary circulation was re-established, protamine sulphate was injected to neutralize the heparin, the chest wall was closed, and post-operative bronchospirometry was performed.

Results Of four sheep in which the operation was

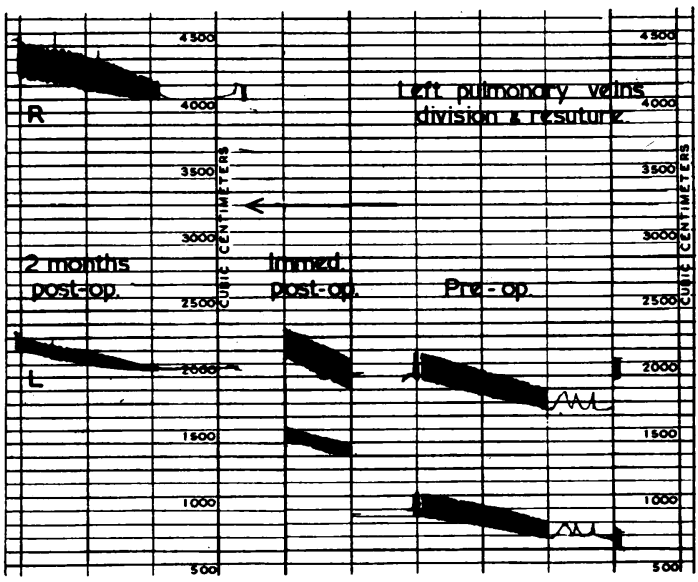

FIG. 13. Differential spirogram showing normal oxygen uptake in the left lung before, after, and two months after division and resuture of the left pulmonary veins.

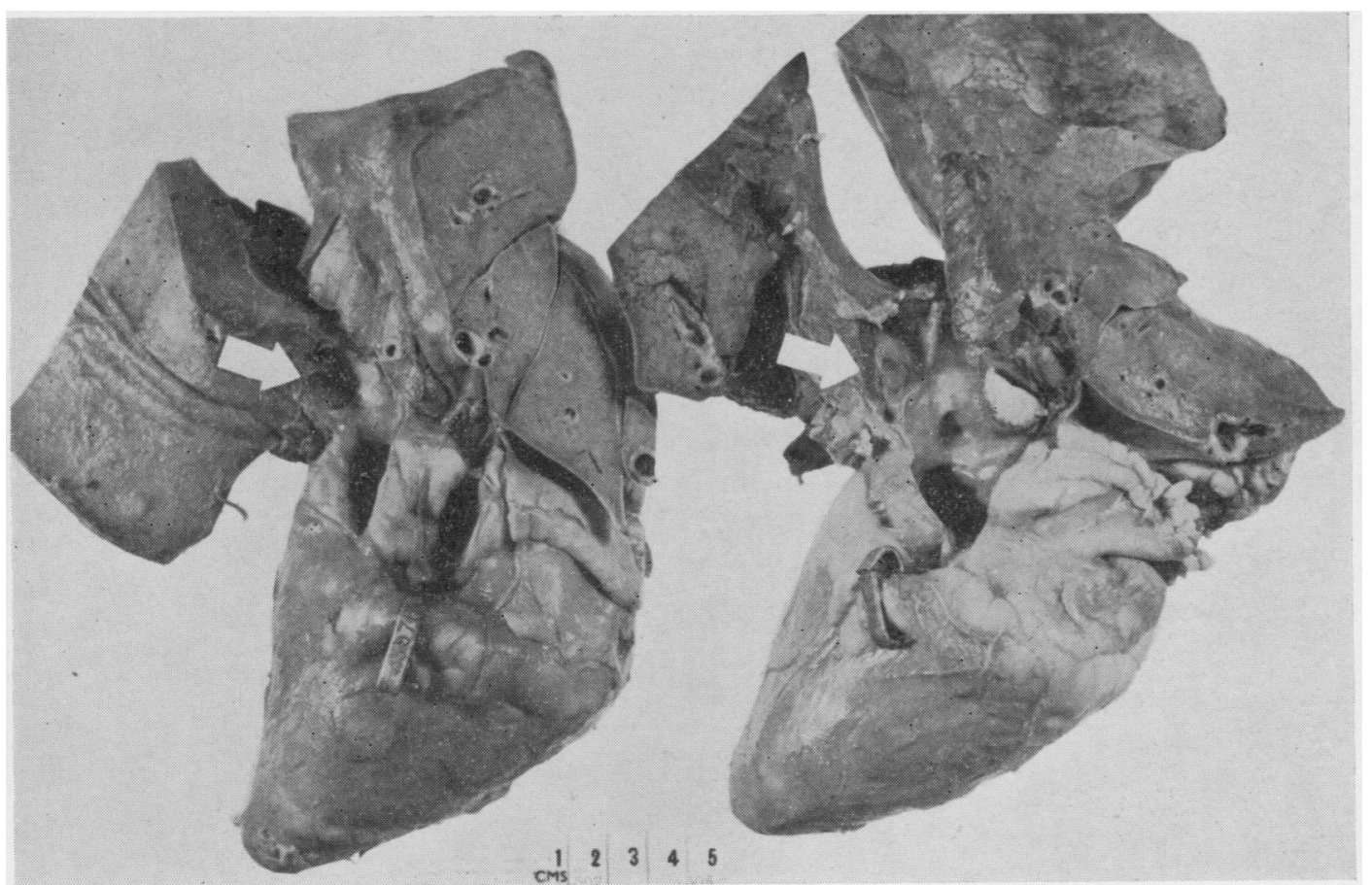

FIG. 14. Macroscopic appearance of two left lungs with normally patent pulmonary veins two months after division and resuture. 

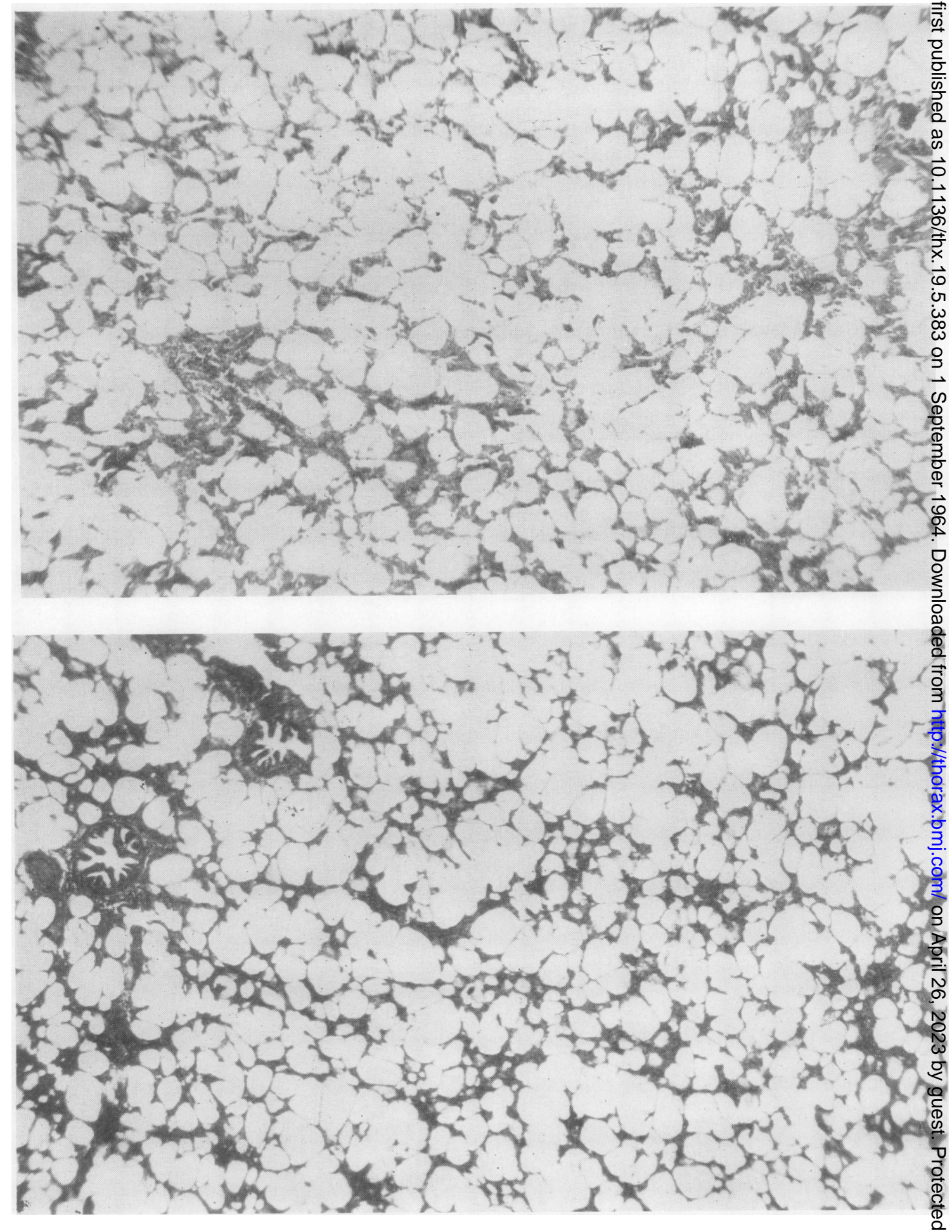

FIG. 15. The normal histological lung pattern of one of these sheep two months after operation: (above) the right lung, (below) the left lung. 
completed, three had normal oxygen uptake three months after operation (Fig. 13). Macroscopically the anastomosis was well healed without any narrowing (Fig. 14). Histologically these lungs were normal (Fig. 15). The fourth animal survived, but at the anastomosis, which was nearer the lung, there was a stricture, and the lung contained a large abscess.

Conclusion Successful pulmonary venous anastomosis, when re-implanting a sheep lung, though difficult, is feasible. The lungs absorb oxygen normally and are histologically normal three months after operation. For success the left pulmonary veins should be divided and resutured as close as possible to the left atrium at the normal site of entry of these veins. This in turn requires a satisfactory atrial clamp and a careful locking suture technique (Borrie and Prapaiwongs, 1960).

\section{TOLERANCE TIME OF SHEEP LUNGS TO ANOXIA}

How long can a lung tolerate total anoxia yet remain viable and function normally? This is a vital question in any lung transplantation experiment or clinical procedure, as an index of the time available for re-establishing pulmonary blood flow.

\section{METHOD}

After differential bronchospirometry and left thoracotomy under normothermia, the pulmonary ligament was divided, the animal heparinized, the bronchial artery ligated, the left main bronchus clamped, and the pulmonary artery and both veins were snared and then firmly occluded.

In successive experiments the anoxic period was increased by quarter-hour intervals from one hour up to four hours.

At the end of each test period the bronchus and vessels were released, the circulation was reestablished, the left lung inflated, and the heparin neutralized with protamine sulphate. The chest wall was closed, and differential spirometry was again performed. In surviving animals, spirometry was repeated two months later, after which the animals were killed and the lungs were examined histologically.

\section{RESULT}

Eighteen experiments were performed. Nine of the animals were subjected to periods of up to two and a half hours of hilar occlusion, and all survived with lungs functioning normally both immediately and two months after operation.

Of the nine animals whose lungs were anoxic for three hours or more, there were three survivors after three, three and a half, and four hours of anoxia respectively. The four-hour survivor developed transient left pulmonary oedema, which was successfully treated by endobronchial aspiration for one hour and tracheostomy for 24 hours. All three had normal oxygen uptake two months, and histologically normal lungs three months after operation.

\section{CONCLUSION}

It is concluded that the left lung of sheep can regularly withstand two and a half hours of anoxia, and that there is no sharp end-point to tolerance of anoxia between three and four hours of circulatory arrest in the test lung. After four hours of anoxia with normothermia pulmonary oedema will regularly occur (Borrie, 1962).

One has therefore at least two hours in hand for transplanting lung, i.e., from the time of arrest of circulation through the lung for transplantation until it is once more receiving blood in its new situation within the host.

\section{LUNG HOMOTRANSPLANTATION}

In 1947, the Russian surgeon Demikhov first transplanted the lower lobe of the right lung of one dog into the lung of another dog, with survival of the recipient for four days. This work only became widely known in 1962 (Demikhov, 1962). That animals can survive after lung transplantation was shown by Hardin and Kittle (1954) who, after transplanting the left lung, immediately performed a right pneumonectomy. Two animals survived for six and nine days, proving unequivocally the functional ability of the homologous lung during these periods.

Experimenters in the 1950s showed that homografts were always rejected and that the recipients usually died within 10 days of operation from pneumonia, the end result of the homograft reaction (Davis, O'Connor, Coloviras, and Strawn, 1952; Neptune et al., 1952).

Attempts to modify this reaction led Neptune, Weller, and Bailey (1953) to give corticotrophin to four dogs with lung homografts, thereby increasing the average survival time to 25 days. One dog survived 42 days. Hardin and Kittle (1954) had survivals from 12 to 18 days after cortisone. They also reported survivals up to 36 days when litter mates were used.

Again, the discovery that antimetabolites and alkylating agents could suppress the immune response in skin and kidney homografts led to their use in experimental lung transplants. 
Blumenstock, Collins, Hechtman, Thomas, and Ferrebee (1962) showed that methotrexate could prolong survival in dogs with a lung homograft up to 410 days, though serial chest films in the long-term survivors showed progressive opacification of the transplanted lung. Hardy, Webb, Dalton, and Walker (1963) found azothioprine (Imuran) to be the most active immuno-suppressant, though their average survival time was only 14 days.

MacPhee and Wright (1964) reported successful lung homografts in four dogs without operative or early post-operative mortality. Cyclophosphamide in combination with other drugs suppressed the immune response for periods up to 49 days. In three dogs, intravascular thrombosis at the atrial suture line was the cause of death.

De Bono and Brock (1964) also briefly reported the survival of dogs up to eight months after lung transplantation, and used methotrexate to suppress the immune response.

Further, the first case report of lung transplantation in man has now been published and, though the patient died 18 days after operation in renal failure, the transplanted lung was functioning well, with no evidence at necropsy of rejection of the graft (Hardy, Eraslam, and Dalton, 1963).

We studied the problems of lung homografts in twin lambs in 1962 and in twin calves in 1963.

\section{HOMOGRAFTS IN TWIN SHEEP}

These lambs, born in the New Zealand spring of 1961 (September), were marked at birth and reared to $100 \mathrm{lb}$. hoggets. Twin sheep, however, are rarely if ever monozygotic. The lung transplantation was completed in four hoggets and served to establish the technique in subsequent lung homografting in twin calves.

The findings showed initial oxygen uptake and good initial air entry by auscultation, the animal at first being fit and feeding normally. Within six days, however, the animals sickened, developed a left pleural effusion, and died on the seventh to ninth post-operative day from bilateral bronchopneumonia. Sectioning of the lungs showed a red, fleshy 'homograft' lung, intact lines of anastomosis, thrombosis in the pulmonary veins arising on the atrial suture line, and pneumonia in the non-grafted lung.

\section{HOMOGRAFTS IN 'IDENTICAL' TWIN CALVES}

Because of the failure with twin sheep, the experiments in 1963 were taken a stage further and performed on animals known to be monozygotic음 identical twins, namely calves.

These animals were obtained by air transport ${ }_{\circ}^{\Phi}$ from the New Zealand Department of Agriculture⿻ Ruakura Animal Research Station, Hamilton,some 600 miles to the north. This station is in the centre of a huge dairy industry based on a cow $\vec{\omega}$ population of over two millions, so that identical sets of twin calves are common, and have been $\vec{x}$ collected and closely studied at Ruakura for over $\rightarrow$ 18 years. This station has a celebrated herd of ${ }_{G}^{\circ}$ identical twin cows covering many researchi projects, so that the station field officers are welle versed in selecting them. The criteria of selection? are set out in the station report Notes on theIdentification of Identical Twin Calves and in $\$$ Hancock's papers (1952).

These include careful appraisal of the physical $\stackrel{\mathbb{\Phi}}{3}$ features including coat colour, the eye-lashes, earo fringes, and tail switch, the head shape including the lower jaw and muzzle, the body conformation, $\vec{\oplus}$ and pigmentation pattern. Particular attention is $\triangleright$ paid to comparing the pigmentation on the ears, $\square$ nose, palate, tongue, lower jaw, lower lip, anovulval area, and udder. In these experiments we did not do confirmatory skin graft tests. Through-ô out we had the utmost co-operation from $\mathrm{Mr}$. $\mathrm{D}$ A. H. Carter, Senior Animal Geneticist, Ruakura Animal Station.

It was appreciated that methotrexate or Imuran might favourably affect the outcome of the experi ments, but the makers of these drugs when approached were unable to deliver supplies because, they said, all that was available was required for kidney transplantation in man.

\section{OPERATIVE TECHNIQUE}

Stage I: The donor With the animal held on its음 haunches, the first twin or donor was anaesthetized with pentobarbitone sodium on the basis of $64 \mathrm{mg}$. 음 to $8 \mathrm{~kg}$. body weight, and $40 \mathrm{mg}$. of suxamethonium dichloride was injected into the external jugular vein. $\mathrm{N}$ The animal was then placed on the operating table, $N$ and the trachea was intubated with a large size Magill endotracheal tube.

With strict aseptic technique a left thoracotomy was performed and the rib retractor was inserted. A no.e 11-gauge French needle connected to a blood-taking $\frac{C}{\mathscr{D}}$ set was next inserted through the pericardium into the left ventricle, and two units of blood were col- $\square$ lected in standard citrate medium. The animal was $\overline{0}$ then heparinized, the trachea was cross-clamped to $\mathbb{D}$ avoid soiling, and the heart and lungs were imme- $\frac{O}{D}$ diately removed.

The left or test lung was next dissected, defining in turn all hilar structures. The left main bronchus was 
lightly clamped and divided at carinal level. The left pulmonary artery was dissected and divided at its point of origin from the main pulmonary artery. Care was taken with the right pulmonary veins which were dissected, ligated near their point of entry into the left atrium, and divided. Thereafter the left lung was again lifted up, and, taking a generous cuff of atrium that included the entry of both the right and left pulmonary veins, the heart was removed.

The left lung thus had a long cuff of left atrium, left pulmonary artery, and left main bronchus. These were trimmed, and the lung, whose vessels contained heparinized blood, was temporarily placed in a sterile dish in a refrigerator at $0^{\circ} \mathrm{C}$.

Stage II: The recipient The second twin was similarly anaesthetized, and a left thoracotomy was performed. The left main bronchus was lightly clamped at carinal level and divided just proximal to its first branch. Similarly, the left pulmonary artery was clamped with a Satinsky clamp and divided. As the pulmonary veins were not required for the reimplantation, their cardiac ends were ligated and divided. The pneumonectomy was thus completed in about 40 minutes from the start of stage I.

In these experiments, the two stages followed each other. Though highly desirable, in our experimental unit it was not possible to perform the two stages simultaneously. This, in turn, may have adversely affected our final results.

Stage III: The transplantation A generous left atrial cuff was taken in a Sellors atrial clamp, and an elliptical segment of atrial wall was removed.

Re-implantation was begun on the venous side by anastomosing the donor atrial cuff to the elliptical atrial incision of the recipient. Blalock continuous everting sutures (0000 black silk) were used throughout with the interrupted locking mattress sutures already described.

The pulmonary artery was next anastomosed by the established technique. The Sellors clamp was then removed from the left atrium, the Satinsky clamp from the left pulmonary artery, and circulation through the transplanted lung was thus re-established. Because of the heparin given immediately before the donor animal was killed, there was little chance of clotting occurring in the capillary system of the donated lung. Immediately the clamps had been released the lung flushed red as blood again entered the pulmonary blood vessels.

The time from taking the donated lung until circulation was re-established through it in the recipient animal was approximately one and a half hours, which is well within the tolerance time of lungs to anoxia.

The chest wall was closed over water-seal drainage which was removed when consciousness returned. In addition, two polythene 'Readivac' catheters were inserted through the chest wall for subsequent aspiration of post-operative pleural effusion with Readivac flasks, sutured to the back, and firmly clamped.

If blood replacement was required this was now given into the external jugular vein using the units of blood previously collected from the twin.

POST-OPERATIVE CARE The animals received careful nursing, the team visiting at least hourly during the day and three-hourly at night. On each occasion the Readivac flask was released and the pleural cavity thus aspirated.

On the first day, up to $100 \mathrm{ml}$. of clear fluid could be aspirated at a time, but this quickly reduced to a few millilitres per day. The Readivac catheters were removed after three to four days.

FUNCTIONING OF THE LUNG Because of the previous long experience with sheep lung implantation, the finding that oxygen uptake was present in the lung at the conclusion of the operation, the knowledge that occasionally animals had succumbed during differential spirometry at the end of the procedure, and the wish to have survivors that could be tested for differential spirometry 20 or more days after operation, in this set of experiments differential spirometry was deliberately omitted at the conclusion of the operation.

RESULTS The operation was completed in three calves.

One died from bronchial stenosis seven days after operation.

Two returned to pasture at the animal farm some two weeks after operation. At that time there was good air entry in the transplanted lung. The animals thrived, gambolled freely in the fields at the farm, and ate greedily.

They were rechecked 55 and 48 days after operation by chest films, bronchoscopy, and necropsy.

Chest films at this stage showed opacification of the grafted lung.

Bronchoscopy showed an occluded left main bronchus half an inch beyond the carina.

Necropsy revealed that in each animal the transplanted lung had become completely rejected and that this test lung, now necrotic, was enclosed in a thick fibrinous pleural cast.

\section{SUMMARY AND CONCLUSIONS}

The technical problems of left lung excision and re-implantation in sheep, followed by left lung transplantation in twin calves have been studied over the past seven years.

A method for performing differential bronchospirometry with standard Carlens tubes and using temporary tracheostomy is described. 
Early experiments on left lung excision and reimplantation in sheep showed that, although the re-implanted lung functioned for a time, ate infarction of the pulmonary vessels occurred. None survived more than six days.

Further analysis by sets of experiments on the left lung showed that (1) ligation of the bronchial artery in the lung did not affect the proportionate oxygen uptake either immediately or within two months of operation; (2) division and resuture of the left pulmonary artery is repetitively feasible without affecting oxygen uptake in the left lung; (3) the left main bronchus can be repetitively divided and rejoined without stenosis using the Blalock everting continuous suture technique combined with interrupted locking sutures; (4) successful pulmonary venous anastomosis is feasible and the lungs absorb oxygen normally and are histologically normal three months after operation, if the left pulmonary veins are divided and resutured as close as possible to the left atrium at the normal site of entry of these veins, provided a satisfactory atrial clamp and a careful everting and locking suture technique are also used; (5) regarding the tolerance time of sheep lungs to anoxia, the left lung can regularly withstand two and a half hours of anoxia. There is no sharp end-point to tolerance of anoxia between three and four hours after circulatory arrest in the test lung. After four hours of anoxia with normothermia irreversible pulmonary oedema will regularly occur.

In lung transplantation in twin lambs, although the lungs functioned at first, the homograft reaction caused death from six to nine days after operation.

In lung transplantation in identical twin calves, the animals survived and had good initial postoperative air entry. Further, when on pasture they thrived. When, however, their test lungs were examined 55 and 48 days after operation, these were found to have been completely rejected by their host and to be encased in a fibrinous pleural cast.

From a biological viewpoint one should be able to transplant successfully identical twin calf lungs, but transplants of skin should also be done as well as relying on surface markings for establishing firm evidence of identical twinning. Immuno suppressants should improve these results.

This work was supported by a grant from the Medica

Research Council of New Zealand.

We gratefully acknowledge encouragement from Professor G. J. Fraenkel, Ralph Barnett Professor oథ Surgery in the University of Otago.

We are indebted to Mr. John Wall, S.R.N. and the staff of the Department of Surgery for technica assistance, to Miss Entwistle for the diagrams, M Gerald Brook for the photographs, and to Miss Richardson for sezretarial help.

\section{REFERENCES}

Alican, F., and Hardy, J. D. (1963). Lung reimplantation. J. Am오. med. Ass., 183, 849.

Blumenstock, D. A., Collins, J. A., Hechtman, H. B., Thomas, E. W. and Ferrebee, J. W. (1962). Functioning homografts of the lu6g

in dogs. Ann. N.Y. Acad. Sci.,99, 882. Sch., 35, 15 .

__ (1962). Tolerance time of sheep lungs to anoxia. Ibid., 40, 5. Campbell, G. D., and Fulton, I. B. (1958). Bronchial arteg division and lung function. Ibid., 36, 31 .

and Foster, E. R.(1959a). Left main bronchus division, resuture

and lung function. Ibid., 37, 20 . insertion of venous homografts. Ibid., 37, 36 . insertion of venous homografts. I. B. (1958). Pulmonary artery division, resuturie and lung function. Ibid., 36, 32 .

and Mitchell, R. M. (1960). The sheep as an experimenQ animal in surgical science. Brit. J. Surg., 47, 435.

and Montgomerie, J. Z. (1958a). Lung excision and reimpla tation in sheep. Proc. Univ. Otago med. Sch., 36, 9.

- (1958b). Differential bronchospirometry in sheep. Ibig 36, 10. Ibig, 36, 10 and Prapaiwongs, T. (1960). Division and resuture of I
pulmonary veins in sheep. Ibid., 38, 18.

Davis, H. A., O'Connor, J. P., Coloviras, G. J., Jr., and Strawn, D. $\overrightarrow{\bar{\delta}}$. (1952). Homologous transplantation of the lung: preliminam report of technical studies. Arch. Surg., 64, 745.

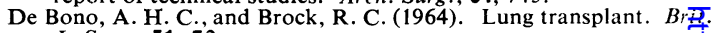
J. Surg., 51, 72.

Demikhov, V. P. (1962). Experimental Transplantation of Vilai Organs. Consultants Bureau, New York.

Gowans, J. L. (1962). In General Pathology, 3rd ed. Edited 局 H. Florey. Lloyd-Luke (Medical Books) Ltd., London.

Hancock, J. (1952). Identical cattle twins and colour inheritan Endeavour, 11, 78

Hardin, C. A., and Kittle, C. F. (1954). Experiences with transplaetation of the lung. Science, 119, 97.

Hardy, J. D., Eraslam, S., and Dalton, M. L., Jr. (1963). Autotrans. plantation and homotransplantation of the lung: further studies. $J$. thorac. cardiovasc. Surg., 46, 606.

- Webb, W. R., Dalton, M. L., Jr., and Walker, G. R., Jr. (1963) Lung homotransplantation in man. J. Amer. med. Ass., 186, 1065 .

Howard, J. G., and Michie, D. (1963). Transplantation immunology In Modern Trends in Immunology, I, p. 226. Edited by R. Cruich shank. Butterworths, London.

Huggins, C. E. (1959). Reimplantation of lobes of the lung. experimental technique. Lancet, 2, 1059.

Juvenelle, A. A., Citret, C., Wiles, C. E., Jr., and Stewart, J. D. (1951). Pneumonectomy with replantation of the lung in the dog for physiologic study. J. thorac. Surg., 21, 111.

MacPhee, I. W., and Wright, E. S. (1964). Experimental lung trans plantation. Lancet, 1, 192 .

Neptune, W. B., Redondo, H. P., and Bailey, C. P. (1952). Expef mental lung transplantation. Surg. Forum, p. 379 Weller, R. W., and Bailey, C. P. (1953). Experimental lup transplantation. J. thorac. Surg., 26, 275.

,

